\title{
Dimensioning of Wireless Mesh Networks with Flow-Level QoS Requirements
}

\author{
Pasi Lassila \\ Helsinki Univ. of Technology \\ P.O.Box 3000, FIN-02015 TKK \\ Finland \\ Pasi.Lassila@tkk.fi
}

\author{
Aleksi Penttinen \\ Helsinki Univ. of Technology \\ P.O.Box 3000, FIN-02015 TKK \\ Finland \\ Aleksi.Penttinen@tkk.fi
}

\author{
Jorma Virtamo \\ Helsinki Univ. of Technology \\ P.O.Box 3000, FIN-02015 TKK \\ Finland \\ Jorma.Virtamo@tkk.fi
}

\begin{abstract}
We study dimensioning of wireless mesh networks with elastic traffic subject to flow-level performance constraints. The objective of the dimensioning is to provide order-of-magnitude estimates of the required transmission resources in the network. The results can be used to evaluate the feasibility and sensitivity of a given network scenario, consisting of node locations, the interference model and the traffic matrix. We apply a network model which incorporates the dynamic flow-level behavior and MAC layer wireless interference. We formulate the dimensioning problems for two capacity parameters resulting from different interference models. Furthermore, three approximation schemes are presented to facilitate the computations. The approaches are illustrated by numerical examples.
\end{abstract}

Categories and Subject Descriptors: C.2.1 [Computer Systems Organization]: Network Architecture and DesignWireless communication; C.4 [Computer Systems Organization]: Performance of systems-Modeling techniques

General Terms: Design, Performance.

Keywords: Dimensioning, wireless networks, flow-level performance, STDMA, balanced fairness.

\section{INTRODUCTION}

Wireless mesh networks (WMNs) are a recent architecture for wireless multihop networks, see [9]. A WMN consists of clients, fixed wireless routers and gateways. The wireless routers form a mesh network, where wireless multi-hop paths are used for communication. Typical applications of WMN technology are wireless neighborhood networks, which provide a cost effective Internet access to their deployment areas by using, for example, WiMAX technology [12].

In this study, we consider dimensioning of WMNs. The task of dimensioning is to assist network planning by providing an order-of-magnitude approximation of the required amount of resources in deploying a network to satisfy given

Permission to make digital or hard copies of all or part of this work for personal or classroom use is granted without fee provided that copies are not made or distributed for profit or commercial advantage and that copies bear this notice and the full citation on the first page. To copy otherwise, to republish, to post on servers or to redistribute to lists, requires prior specific permission and/or a fee.

PE-WASUN'06, October 6, 2006, Torremolinos, Malaga, Spain.

Copyright 2006 ACM 1-59593-487-1/06/0010 ...\$5.00. traffic and QoS requirements. Dimensioning of WMNs is a difficult task due to the complex wireless interference phenomena that affect the system capacity. Additionally, since much of the traffic in a WMN-based Internet access network is traditional data traffic, the QoS requirements used in dimensioning can be reasonably defined only at the flow level, which adds another layer of complexity. Flow-level QoS requirements are defined for example in terms of per flow throughput or file transfer delay.

As the main contribution of this paper we formulate dimensioning problems for WMNs taking into account both the interference and the flow-level performance. In more detail, the input parameters of the dimensioning problems are the locations of the wireless routers, the routes used by the flows, and the offered traffic loads and the target throughput requirements on these routes. The output from the problem is an estimate of the required amount of resources. To achieve simple order-of-magnitude estimates of the resources, we describe the resources in each interference model by a single parameter. This parameter reflects whether or not a given network scenario has a feasible implementation.

We assume that the WMN MAC layer can be reasonably modeled by STDMA [18]. In STDMA, sets of links that can transmit simultaneously (so-called transmission modes) are scheduled in a TDMA manner. Although the STDMA approach can incorporate almost any kind of interference model, we concentrate on two specific models for which the resources can be easily parameterized.

The Boolean interference model assumes that interference may restrict which links can be active simultaneously, but does not affect the active link capacities. In other words, all active links have the same constant capacity. Within the Boolean model, transmission mode feasibility could be determined by different rules. An interference rule might be, for example, that if a node receiving a transmission on a link lies within the transmission range of the sender of some other active link the two links cannot exist in the same transmission mode. For the Boolean model the set of transmission modes consists of all permitted combinations of active links (which are not itself subsets of other modes). In this model, the capacity parameter is the scaling factor which scales all the link capacities. The scaling factor represents the nominal data rate of the wireless routers.

The Shannon interference model assumes that also the link capacities are affected by interfering transmissions. We assume an ideal link adaptation, i.e., the link capacities are 
determined by Shannon's information theoretic bound. Under STDMA it is advantageous for the links to use maximum transmission power when active and refrain from further power adaptation, cf. [22]. In this case the set of all transmission modes consists of all combinations of active links, but the set can also be truncated by Boolean type interference rules. We assume that the routers have the same maximum transmission power which acts also as the capacity parameter of the model.

The dimensioning is based on flow-level QoS requirements. As a consequence of STDMA operating on a fast time scale, the flows, such as file transfers, observe the network as if it had fixed link capacities. By changing the STDMA schedule we may transfer capacity from one part of the network to another within certain limits. Thus, the resources available to the flows are defined by the scheduling. However, the flow-level performance depends also on how the flows share the resources. Data traffic shares the available bandwidth fairly, e.g., by TCP protocol. This has a significant impact on the flow level performance. For example, the throughput of a file transfer does not remain constant during the transfer. As new flows arrive and existing flows depart from the network, also the resource allocation changes accordingly. In order to characterize the flow level performance, one must account for the dynamic nature of flows.

In this paper, we approximate the resource sharing policy by balanced fairness and express the QoS requirements as average throughputs on the given routes. Balanced fairness is a resource sharing notion developed by Bonald and Proutière [5]. It allows us to characterize (either directly or with suitable bounds) the average throughput in a robust manner, because the throughput characteristics depend on the traffic only through the average traffic load on each route. In other words, no additional information is required about the traffic to account for the flow-level QoS requirements than what is already necessary for basic dimensioning. Furthermore, balanced fairness is known to often approximate well other bandwidth sharing schemes [3].

The resulting dimensioning tasks can be viewed as crosslayer optimization problems, where both the MAC layer schedule and the flow-level performance are optimized simultaneously. To overcome the computational complexity involved in non-linear cross-layer optimization, we also develop approximation schemes based on problem decomposition. Notably, in the simplest approximation the dimensioning reduces to an LP problem. The various methods are illustrated through numerical examples.

The paper is organized as follows. Section 2 discusses the assumptions for our dimensioning problems and the network model. The problem formulations for the Boolean model and the Shannon model are given in Sections 3 and approximation schemes are considered in Section 4. Numerical examples are provided in Section 5. We conclude in Section 6 .

\subsection{Related work}

Dimensioning is a classical teletraffic problem which is well understood in traditional circuit switched networks (cf. the use of the Erlang formula). Dimensioning of data networks has been studied to a lesser extent. Well-known methods for this include the square-root method based on packet delay models of $\mathrm{M} / \mathrm{M} / 1$ queuing networks [16] and the use of multi-commodity flow optimization models [21]. Recently, also flow-level models have been applied, for example single link dimensioning is considered in [4] and complete network dimensioning in [17].

Dimensioning has not been much investigated in the context of wireless multi-hop networks. However, dimensioning is closely related to capacity modeling of wireless multihop systems, where the most important aspect to adequately capture is the impact of interference. In the seminal paper by Gupta and Kumar [11], it has been shown that the per node throughput scales with the number of nodes $n$ as $\mathrm{O}(\sqrt{n})$. Basically, the work in [11] and a number of subsequent generalizations, such as [10], consider random networks with greedy sources under asymptotic settings, which provide significant insights into the fundamental tradeoffs in wireless networking.

Another large body of work considers the performance of a given static wireless network. This case is also more relevant to WMNs as they are assumed to consist of stationary wireless routers. When modeling the performance of a fixed wireless network, the impact of interference can be taken into account by for example determining the sets of links that interfere with each other. A simple model following this approach is given by Jun and Sichitu [15]. However, the model gives somewhat pessimistic results due to the very stringent interference assumptions. Jain et al. [13] develop a more elaborate model that allows one to iteratively construct increasingly accurate lower and upper bounds for the capacity under varying assumptions on the physical reasons causing the interference.

Another approach entails assuming that the network operates according STDMA as introduced in [18], which specifically assumes that interfering links are scheduled in a TDMA manner. The schedule can then be optimized to maximize the system capacity, cf. [1] and [14].

The flow-level performance of wireless networks has been analyzed in [19, 20] and [3]. These studies generalize the notion of balanced fairness, which was introduced for analyzing fixed networks by Bonald and Proutière [5], to wireless networks. The models determine the flow-level performance while simultaneously optimizing performance on the MAC layer, which is assumed to operate according to STDMA.

Many of the results cited above could in principle be used for dimensioning, but the models are often numerically too complex for realistic network sizes. In this paper we utilize some of the results related to balanced fairness and present simple numerically tractable optimization formulations for the dimensioning task that account for both the wireless interferences and the flow-level QoS requirements.

\section{WIRELESS NETWORK MODEL WITH ELASTIC TRAFFIC}

\subsection{Interference modeling in STDMA}

The network consists of a set of nodes connected by unidirectional links $\mathcal{L}=\{1, \ldots, L\}$. The existence of a link and its capacity between a given pair of nodes depends on the assumed interference model. When assuming STDMA as the MAC layer protocol, the link capacities are characterized by the so-called set of transmission modes. Each transmission mode contains information about which links can operate simultaneously and their respective capacities, and one needs to enumerate all such link combinations. The number of transmission modes is denoted by $M$. The set of 


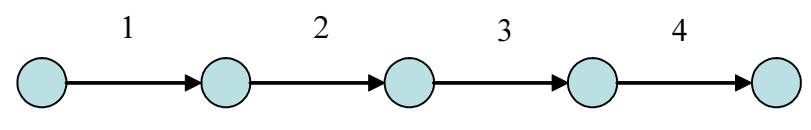

Figure 1: Linear network with five nodes and four links.

transmission modes is represented by the matrix $\mathbf{S}$

$$
\mathbf{S}=\left(\begin{array}{ccc}
c_{11} & \cdots & c_{1 M} \\
\vdots & \ddots & \vdots \\
c_{L 1} & \cdots & c_{L M}
\end{array}\right)
$$

where $c_{l m}$ denotes the capacity of link $l$ in transmission mode $m$. Note that $c_{l m}=0$ when link $l$ is not used in mode $m$. Associated with the transmission modes is a schedule, defined as the vector $\mathbf{t}=\left[t_{1}, \ldots, t_{M}\right]^{\mathrm{T}}$, where $t_{m}$ gives the proportion of time transmission mode $m$ is used. As a notational convention, we assume that vectors are always column vectors. Thus, the components of $\mathbf{t}$ satisfy $\mathbf{e}^{\mathrm{T}} \mathbf{t}=1$, where $\mathbf{e}$ is an $M$-dimensional vector with all elements equal to one, i.e., $\mathbf{e}=[1, \ldots, 1]^{\mathrm{T}}$. In this work, we assume that the network operates using a fixed schedule. Note that the capacity of the system depends on the choice of the schedule $\mathbf{t}$, and it allows us to optimize the capacity usage of the wireless network with respect to the schedule.

In this paper we consider the use of two interference models: the Boolean model and the Shannon model.

\subsubsection{Boolean model}

In the Boolean model, it is assumed that the link capacity is a constant, denoted by $b$, within the communication range. Hence, each $c_{l m}$ in $\mathbf{S}$ is equal to either 0 or $b$ according to the interference rules. As an example, consider the case where each node is equipped with a single radio. In this case the interference rule is that a node cannot transmit and receive simultaneously. Additionally, secondary conflicts could be modeled by an interference rule which states, e.g., that there can be only one transmitting node within the communication range of any receiver (i.e., it is assumed that all nodes have the same communications range). In a linear network with five nodes and four uni-directional links shown in Figure 1, where each node has exactly one receiver node within its communication range, there are the following three transmission modes,

$$
\mathbf{S}=b \cdot\left(\begin{array}{ccc}
1 & 0 & 0 \\
0 & 1 & 0 \\
0 & 0 & 1 \\
1 & 0 & 0
\end{array}\right)
$$

\subsubsection{Shannon model}

In the Shannon model, the link capacities are based on the signal-to-interference-and-noise ratios (SINR) at the nodes and we approximate the capacity of the link with Shannon's information theoretic bound

$$
c=w \cdot \log _{2}(1+\mathrm{SINR}),
$$

where $w$ denotes the bandwidth of the channel. SINR depends on the set of other active links. We denote the set of active links in transmission mode $m$ by $\tau_{m}$, i.e., $\tau_{m}=\{l \in$
$\left.\mathcal{L}: c_{l m} \neq 0\right\}$ We assume that the noise power $\nu$ is constant for all links and that all active nodes transmit at the maximum transmission power $p$. The elements of the capacity matrix $\mathbf{S}$ are given by

$$
c_{l m}=w \cdot \log _{2}\left(1+\frac{g_{l l}}{\sum_{j \in \tau_{m}} g_{j l}+\nu / p}\right),
$$

where $g_{j l}$ denotes the power gain between the transmitter of link $j$ and the receiver of link $l$ and is assumed to be given by $g_{i j}=d_{i j}^{-\alpha}$. Here $d_{i j}$ is the distance between the transmitter of link $i$ and the receiver of link $j$ and the exponent $\alpha$ reflects the attenuation characteristics of the signal. In the numerical examples we set $\alpha=4$.

In the worst case there are $2^{L}-1$ different transmission modes. However, due to physical constraints (e.g., nodes have only a single radio) this number can be substantially smaller. Referring back to the linear network example in Figure 1, if it is assumed that each node has a single radio, the capacity matrix $\mathbf{S}$ under the Shannon model becomes

$$
\mathbf{S}(p)=\left(\begin{array}{ccccccc}
c_{11} & 0 & 0 & 0 & c_{15} & 0 & c_{17} \\
0 & c_{22} & 0 & 0 & 0 & c_{26} & 0 \\
0 & 0 & c_{33} & 0 & c_{35} & 0 & 0 \\
0 & 0 & 0 & c_{44} & 0 & c_{46} & c_{47}
\end{array}\right)
$$

\subsection{Performance under balanced fairness}

The traffic in the network is routed along $N$ routes, i.e., there are $N$ classes of flows in the system, and the set of flow classes is denoted by $\mathcal{F}=\{1, \ldots, N\}$. The routing is described by the matrix $\mathbf{A}$, where the element $a_{l i}=1$ if link $l$ is used by class- $i$ traffic and 0 otherwise. We additionally denote the set of links used by class $i$ as $\mathcal{R}_{i}$. When link $l$ is on route $\mathcal{R}_{i}$ we use the notation $l \in \mathcal{R}_{i}$.

The performance of the network is evaluated at the flow level, where flows arrive and depart randomly, and the flows are assumed to share the bandwidth dynamically according to balanced fairness. Due to the insensitivity property, the flow-level performance depends only on the load of class- $i$ flows $\rho_{i}$ given by the product of the mean arrival rate of class- $i$ flows and their mean flow size. Thus, load $\rho_{i}$ is a measure of the mean volume of information offered by flows in class $i$ per unit time. The vector of loads in all classes is denoted by $\boldsymbol{\rho}=\left[\rho_{1}, \ldots, \rho_{K}\right]^{\mathrm{T}}$. Also, we denote the vector of offered loads on each link by $\mathbf{r}$ and it is given by $\mathbf{r}=\mathbf{A} \boldsymbol{\rho}$.

In the context of fixed networks with a link capacity vector c, the performance at the flow level under balanced fairness, i.e., throughput or mean flow (file) transfer delay, can be given an explicit expression for some special network topologies, see [6]. For multi-level trees, which is a common topology in access networks, the performance can be obtained quite efficiently using the recursive formulae in [8]. For general topologies, the exact computation becomes computationally quite heavy, and thus impractical for dimensioning purposes. However, there are also simple and explicit approximations available for the performance.

The most straightforward approximation to the throughput of class- $i$ flows is to approximate the throughput by the available capacity of the bottleneck link along the route of the class. Thus, the throughput function $\Gamma_{i}(\mathbf{c})$ is given by

$$
\Gamma_{i}(\mathbf{c})=\left(\max _{l \in \mathcal{R}_{i}} \frac{1}{c_{l}-r_{l}}\right)^{-1},
$$

where $\mathbf{c}$ denotes the vector of link capacities and $c_{i}$ its $i$ th 
component. Similarly, $r_{i}$ is the $i$ th component of the link load vector $\mathbf{r}$. In fact, (2) provides an upper bound on the performance, as shown in [6], and it is accurate when the performance of the class is mostly determined by a single bottleneck. However, a very tight lower bound for the throughput has been recently derived by Bonald [2]. The corresponding throughput function $\Gamma_{i}(\mathbf{c})$ is

$$
\Gamma_{i}(\mathbf{c})=\left(\max _{l \in \mathcal{R}_{i}} \frac{1}{c_{l}}+\sum_{l \in \mathcal{R}_{i}} \frac{r_{l}}{c_{l}} \frac{1}{c_{l}-r_{l}}\right)^{-1} .
$$

The above formulae also provide insight into when detailed flow-level dimensioning is useful. For example, from (3) it can be seen that throughput depends on additive contributions from each link along the route of the class. The contributions from high speed core links even with modest overprovisioning are negligible compared with the contributions due to the access networks. Thus, detailed flow-level dimensioning is required only for access networks, such as realized by WMNs.

In our approach, the link capacity matrix $\mathbf{S}$ and the schedule $\mathbf{t}$ jointly determine the link capacities of the WMN. Given the schedule $\mathbf{t}$, the effective bandwidths of the links available at the flow level are given by

$$
\mathbf{c}=\mathbf{S t},
$$

i.e., the effective bandwidth of link $l$ simply equals the sum of the capacities of link $l$ in the $M$ modes weighted with the corresponding time proportions of the schedule $\mathbf{t}$. Thus, under balanced fairness the network with fixed STDMA scheduling corresponds to a fixed network with the capacity vector c given by (4). By choosing the schedule appropriately we may optimize the resource usage with respect to the flowlevel performance, and this is utilized in our dimensioning formulations.

\section{PROBLEM FORMULATIONS}

We take into account the fact that the traffic is dynamic consisting of randomly arriving flows. The flows share the bandwidth according to balanced fairness. The wireless network MAC layer is realized by STDMA with an optimized, fixed schedule. The input parameters for the dimensioning task are the locations of the wireless routers, the routes in the network and the traffic parameters of each route (i.e., the offered load and the respective throughput requirement).

We describe the required resources by a capacity parameter. We find the minimum value of the parameter that is sufficient to satisfy the throughput requirements when the resource usage as determined by the scheduling is optimized simultaneously. In the following, two interference models are used resulting in two different capacity parameters.

\subsection{Dimensioning with Boolean model}

We first consider the case where the link capacities are modeled according to the simple Boolean model and we assume that all routers have the same radio with traffic capacity of $b$ bit/s. Then the task is to determine a minimal nominal capacity of the radio $b$ that is sufficient to have a pre-defined performance level under a given traffic load. This problem can be viewed as a first approximation to dimensioning, where the objective is to get a rough idea of how much traffic handling capacity the network needs.

\subsubsection{Formulation for capacity limit}

The most straightforward approach to dimensioning of a WMN would be to define the dimensioning problem in terms of the maximum sustainable load and ignore the flow-level performance. This corresponds to dimensioning based on the so called capacity limit, which bounds the stability region. Thus, the optimization problem for the capacity parameter $b$ can be expressed as

$$
\begin{array}{r}
\min _{b, \mathbf{t}} b \\
b \mathbf{M t} \geq \mathbf{r}, \\
\mathbf{e}^{\mathrm{T}} \mathbf{t}=1, \\
\mathbf{t} \geq 0,
\end{array}
$$

where we have decomposed the capacity matrix as $\mathbf{S}=$ $b \mathbf{M}$. Therefore, $\mathbf{M}$ is an indicator matrix with each element equaling 0 or 1 depending on whether the link is active in the corresponding mode or not. The first constraint reflects the stability of the dynamic system; the system is stable if $c_{l}>r_{l}, \forall l$.

The above problem can be cast into an equivalent LP (linear programming) form by making the variable substitution $q_{m}=b t_{m}$. Let $\mathbf{q}$ denote the vector with the new variables $q_{m}$, i.e., $\mathbf{q}=\left[q_{1}, \ldots, q_{M}\right]^{\mathrm{T}}$. Now $\mathbf{e}^{\mathrm{T}} \mathbf{q}=b$ and the optimization problem can be becomes

$$
\begin{aligned}
b= & \min _{\mathbf{q}} \mathbf{e}^{\mathrm{T}} \mathbf{q}, \\
& \mathbf{M q} \geq \mathbf{r}, \\
& \mathbf{q} \geq 0 .
\end{aligned}
$$

\subsubsection{Formulation with throughput requirements}

While the capacity limit approach is meaningful from the system point of view, it does not provide any information about the QoS experienced by the users. We use the perflow throughput, given by $\Gamma_{i}(\mathbf{S t})$, as the QoS criterion and for each class the throughput requirement is denoted by $\gamma_{i}$. As the throughput has a meaningful interpretation only in a stable system, we set $\Gamma_{i}(\mathbf{c})=0$ if $\exists l: c_{l} \leq r_{l}$, i.e., if the capacity vector lies outside the stability region. Thus, the flow-level constraints replace the stability constraint in (6) and the optimization problem can be expressed as

$$
\begin{aligned}
b= & \min _{\mathbf{q}} \mathbf{e}^{\mathrm{T}} \mathbf{q}, \\
& \Gamma_{i}(\mathbf{M} \mathbf{q}) \geq \gamma_{i}, \quad \forall i, \\
& \mathbf{q} \geq 0 .
\end{aligned}
$$

Even with the simplest approximation for the throughput, cf. (2), the problem is non-linear and computationally difficult for large problem instances. Later, in Section 4, we consider approximative approaches that can be solved numerically more efficiently.

\subsection{Dimensioning with Shannon model}

Next we consider the use of the more detailed capacity modeling based on (1). We assume that the wireless routers have a common maximum transmission power, denoted by $p$. This is our capacity parameter, i.e., we find the minimum $p$ required to have a sufficient performance level under a given traffic scenario.

\subsubsection{Formulation for capacity limit}

Again, we first consider dimensioning based on the capacity limit. To emphasize that the link capacities depend 
on the power $p$, the capacity matrix is expressed as $\mathbf{S}(p)$, and the optimization problem for the capacity parameter $p$ becomes

$$
\begin{aligned}
\min _{p, \mathbf{t}} p & \\
\mathbf{S}(p) \mathbf{t} & \geq \mathbf{r}, \\
\mathbf{e}^{\mathrm{T}} \mathbf{t} & =1, \\
\mathbf{t} & \geq 0 .
\end{aligned}
$$

Unlike in the case of the Boolean capacity model, the above cannot be converted into an LP problem due to the complex non-linear representation of the link capacities.

However, it is still possible to solve the problem efficiently. For a given $p$ the elements of the capacity matrix $\mathbf{S}(p)$ have fixed values and the problem becomes essentially a similar problem of determining a minimum capacity scaling factor as in (5). Thus, the problem can be expressed as

$$
\begin{aligned}
f(p)= & \min _{\mathbf{q}} \mathbf{e}^{\mathrm{T}} \mathbf{q}, \\
& \mathbf{S}(p) \mathbf{q} \geq \mathbf{r}, \\
& \mathbf{q} \geq 0 .
\end{aligned}
$$

As the link capacities are monotonous with respect to $p$, the minimum required $p$ to satisfy the stability constraints is given by

$$
p: f(p)=1 \text {. }
$$

i.e., when the minimum required scaling is equal to one. At this point, there exists a schedule under which the link capacities are just large enough to satisfy the stability constraints. Thus, the optimum $p$ is found by a line search over the solutions to the LP problem (7).

\subsubsection{Formulation with flow-level QoS}

Similarly as before we replace the stability constraints with the more stringent throughput requirements. Using the same formulation as above in (7) and (8) the optimization problem can be given as

$$
\begin{aligned}
f(p)= & \min _{\mathbf{q}} \mathbf{e}^{\mathrm{T}} \mathbf{q}, \\
& \Gamma_{i}(\mathbf{S}(p) \mathbf{q}) \geq \gamma_{i}, \quad \forall i, \\
& \mathbf{q} \geq 0 .
\end{aligned}
$$

The optimal solution for $p$ is then solved from

$$
p: f(p)=1 \text {. }
$$

Note that the problem (9) is not an LP problem due to the non-linear nature of throughput functions. Again, this may cause computational problems for large networks and approximate methods may be needed.

\section{APPROXIMATION METHODS}

The large number of the transmission modes and the nonlinear flow-level performance constraints make the problem computationally intractable for large instances. Hence, approximation methods are needed.

\subsection{LP approximation}

The LP approximation is based on the upper bound of the throughput as given by (2). That is, we assume that only the bottleneck link on each route has an effect on the throughput. This approximation gives us the following constraints (given that the feasibility constraint $\mathbf{c} \geq \mathbf{r}$ is satisfied)

$$
\left(\max _{l \in \mathcal{R}_{i}} \frac{1}{c_{l}-r_{l}}\right)^{-1} \geq \gamma_{i}, \quad \forall i .
$$

Since the constraint holds for the maximum value, it must hold also for all the links in the group. Thus,

$$
\frac{1}{c_{l}-r_{l}} \leq \gamma_{i}^{-1}, \quad \forall l \in \mathcal{R}_{i}, \forall i
$$

Denote by $\varphi_{l}=\max _{i}\left\{\gamma_{i}: a_{l i}=1\right\}$ for all $l$, i.e., the maximum throughput requirement on each link. Considering only the most stringent constraints, we may write

$$
c_{l} \geq r_{l}+\varphi_{l} .
$$

In other words, $\varphi_{l}$ represents an optimistic estimate of the additional capacity needed to satisfy the throughput requirements on link $l$.

For the Boolean model, the dimensioning problem is

$$
\begin{aligned}
b= & \min _{\mathbf{q}} \mathbf{e}^{\mathrm{T}} \mathbf{q}, \\
& \mathbf{S q} \geq \mathbf{r}+\boldsymbol{\varphi} \\
& \mathbf{q} \geq 0
\end{aligned}
$$

Note that the above formulation is entirely an LP problem. In the Shannon model, we determine the capacity parameter which satisfies $p: f(p)=1$, where

$$
\begin{aligned}
f(p)= & \min _{\mathbf{q}} \mathbf{e}^{\mathrm{T}} \mathbf{q}, \\
& \mathbf{S}(p) \mathbf{q} \geq \mathbf{r}+\boldsymbol{\varphi}, \\
& \mathbf{q} \geq 0 .
\end{aligned}
$$

\subsection{Fixed schedule approach}

The idea of this approach is to first choose a fixed schedule and then scale the link capacities until the throughput requirements are satisfied. Ideally the chosen schedule would resemble the schedule obtained from the joint optimization of the schedule and the capacity parameter, cf. Section 3. A reasonable approximation to the optimal schedule may be obtained simply by using the solution of the LP approximations presented in Section 4.1. Another intuitive choice would be to use the schedule as determined by the capacity limit formulations.

Given the fixed schedule $\mathbf{t}^{*}$ the capacity of the links is scaled appropriately. For the Boolean model one solves

$$
\begin{aligned}
b= & \arg \min _{b} \\
& \Gamma_{i}\left(b \mathbf{M} \mathbf{t}^{*}\right) \geq \gamma_{i}, \quad \forall i .
\end{aligned}
$$

For the Shannon model the problem is

$$
\begin{aligned}
p= & \arg \min _{p} \\
& \Gamma_{i}\left(\mathbf{S}(p) \mathbf{t}^{*}\right) \geq \gamma_{i}, \quad \forall i .
\end{aligned}
$$

In the above formulations $\Gamma_{i}$ represents either the exact throughput expression for the system under balanced fairness or a suitable bound, for example (3). Note that in the Shannon model this approach may not have a feasible solution. Given a fixed set of modes defined by the schedule $\mathbf{t}^{*}$ there may exist a link the capacity of which cannot be increased sufficiently to meet the throughput demands. This is a consequence of (1) having a limiting value as $p \rightarrow \infty$, when the corresponding mode contains more than one link. 


\subsection{Fixed capacity vector approach}

In this approach we first determine a link capacity vector which is feasible for the given flow level constraints and optimal with respect to some simple criteria. This capacity vector is then realized by scheduling, cf. Section 4.1.

One of the most straightforward objectives to produce a capacity vector $\mathbf{c}$ without scheduling information is to minimize the sum of the link capacities. Assume the storeand-forward bound [6] for the throughput, for which the throughput function is given by

$$
\Gamma_{i}(\mathbf{c})=\left(\sum_{l \in \mathcal{R}_{i}} d_{l}^{-1}\right)^{-1},
$$

where $d_{l}=c_{l}-r_{l}$, we obtain the following optimization problem

$$
\begin{aligned}
& \min _{\mathbf{d}} \mathbf{e}^{\mathrm{T}} \mathbf{d} \\
& \sum_{l \in \mathcal{R}_{i}} d_{l}^{-1} \leq \gamma_{i}^{-1}, \quad \forall i, \\
& \mathbf{d}>0 .
\end{aligned}
$$

Instead of solving this problem directly, it is more convenient to examine the corresponding dual problem. The Lagrangian dual to the problem becomes

$$
\begin{aligned}
& \max _{u} \Theta(u)=\sum_{l} 2 \sqrt{U_{l}}-\sum_{i} \frac{u_{i}}{\gamma_{i}}, \\
& u \geq 0,
\end{aligned}
$$

where $U_{l}=\sum_{i: a_{l i}=1} u_{i}$. Given the solution $\hat{\mathbf{u}}$, the optimal capacity vector to (11) equals

$$
c_{l}=\sqrt{\hat{U}_{l}}+r_{l}
$$

Note that if all the throughput constraints are binding, the dual problem corresponds to solving the equations

$$
\sum_{l \in \mathcal{R}_{i}} U_{l}^{-\frac{1}{2}}=\gamma_{i}^{-1}, \quad \forall i .
$$

In general, there may be also redundant constraints. For these the respective $u_{i}=0$, but for which indices $i$ this holds is not obvious. However, one can always solve directly the dual problem (12), which is considerably simpler than the original full formulations in Section 3. With the capacities c, as given by (13), the scheduling problem is as in the LPapproximation approach earlier by replacing $\mathbf{r}+\boldsymbol{\varphi}$ with $\mathbf{c}$.

As the capacity vector is here computed according to a conservative lower bound for the throughput, we may further improve the result by downscaling the capacity to the point where a tighter bound, such as (3), becomes violated. In principle, any fixed network dimensioning method could be used to produce a feasible capacity vector for this approach.

\section{NUMERICAL EXAMPLES}

\subsection{Simple example with two links}

To illustrate the use of different approximation schemes for dimensioning, we consider first the simple example shown in Figure 2. There are two flow classes (routes) and two links. We assume that middle node B cannot transmit and receive simultaneously, which prevents the concurrent

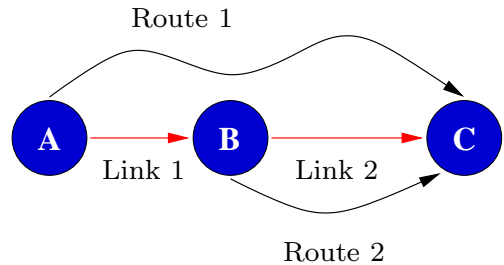

Figure 2: Illustration of the two link example.

use of the links. The dimensioning parameters are $\boldsymbol{\rho}=$ $[0.2,0.3]^{\mathrm{T}} \mathrm{Mbps}, \boldsymbol{\gamma}=[0.1,0.2]^{\mathrm{T}} \mathrm{Mbps}$, with the routing

$$
\mathbf{A}=\left(\begin{array}{ll}
1 & 0 \\
1 & 1
\end{array}\right)
$$

The link loads are $\mathbf{r}=\mathbf{A} \boldsymbol{\rho}=[0.2,0.5]^{\mathrm{T}}$ Mbps.

The network in our example corresponds to a so-called parking lot configuration, for which the exact throughput formulae under balanced fairness are known [7],

$$
\begin{aligned}
& \Gamma_{1}^{\mathrm{OPT}}(\mathbf{c})=\left(\frac{1}{c_{1}-r_{1}}+\frac{1}{c_{2}-r_{2}}-\frac{1}{c_{2}-r_{1}}\right)^{-1}, \\
& \Gamma_{2}^{\mathrm{OPT}}(\mathbf{c})=c_{2}-r_{2} .
\end{aligned}
$$

Recall that with the assumption of a fixed schedule, the schedule is determined by $\mathbf{t}=[t,(1-t)]^{\mathrm{T}}$ and the link capacities are given by $\mathbf{c}=\mathbf{S t}$.

In the approximations we consider the improved storeand-forward bound (ISF) which defines the feasible rate of the system in the stable region $\mathbf{c}>\mathbf{r}$ as follows

$$
\begin{aligned}
& \Gamma_{1}^{\mathrm{ISF}}(\mathbf{c})= \\
& \quad\left(\frac{1}{\min \left(c_{1}, c_{2}\right)}+\frac{r_{1}}{c_{1}\left(c_{1}-r_{1}\right)}+\frac{r_{2}}{c_{2}\left(c_{2}-r_{2}\right)}\right)^{-1}, \\
& \Gamma_{2}^{\mathrm{ISF}}(\mathbf{c})=c_{2}-r_{2} .
\end{aligned}
$$

Note that for class 2 the ISF bound equals the exact solution.

\subsubsection{Dimensioning with the Boolean model}

First, we consider the system with link capacities according to the Boolean model. The transmission mode matrix is then

$$
\mathbf{M}=\left(\begin{array}{ll}
1 & 0 \\
0 & 1
\end{array}\right)
$$

i.e., the links have equal transmission rates but they cannot be active simultaneously. The capacity limit for this system is $b_{\mathrm{CL}}=r_{1}+r_{2}=0.7 \mathrm{Mbps}$.

The optimal solution is obtained from

$$
\begin{aligned}
b_{\mathrm{OPT}}= & \min _{t, b} b \\
& \Gamma_{1}^{\mathrm{OPT}}\left([t b,(1-t) b]^{\mathrm{T}}\right) \geq \gamma_{1}, \\
& \Gamma_{2}^{\mathrm{OPT}}\left([t b,(1-t) b]^{\mathrm{T}}\right) \geq \gamma_{2} .
\end{aligned}
$$

The solution is computed numerically.

The LP approximation gives an optimistic value to the required capacity. The problem is formulated as

$$
\begin{aligned}
b_{\mathrm{LP}}= & \min q_{1}+q_{2} \\
& q_{1} \geq r_{1}+\gamma_{1}, \\
& q_{2} \geq r_{2}+\gamma_{2}, \\
& q_{1}, q_{2} \geq 0,
\end{aligned}
$$


where the second constraint follows from $\gamma_{2}>\gamma_{1}$. Clearly, the solution is $b_{\mathrm{LP}}=r_{1}+r_{2}+\gamma_{1}+\gamma_{2}$.

The optimum of the LP-approximation is realized by the schedule $\mathbf{t}^{*}=[0.3,0.7]^{\mathrm{T}}$. We utilize this schedule in the fixed schedule approach (FS). That is, we conduct a line search with respect to the capacity parameter $b_{\mathrm{FS}}$

$$
\begin{aligned}
b_{\mathrm{FS}}= & \min _{b} b \\
& \Gamma_{1}^{\mathrm{ISF}}\left(b \mathbf{M} \mathbf{t}^{*}\right) \geq \gamma_{1}, \\
& \Gamma_{2}^{\mathrm{ISF}}\left(b \mathbf{M} \mathbf{t}^{*}\right) \geq \gamma_{2} .
\end{aligned}
$$

Note that in this particular case one could use $\Gamma_{i}^{\text {OPT }}$, i.e., the exact throughput formulae, instead of $\Gamma_{i}^{\mathrm{ISF}}$.

Finally, the fixed capacity approach (FC) is used. We construct a feasible capacity vector by solving $\min \sum_{l} d_{l}$ with respect to the $\mathrm{SF}$ bound, cf. Section 4.3. Since $\gamma_{2}>\gamma_{1}$, we obtain the solution by solving

$$
\left\{\begin{array}{l}
\frac{1}{\sqrt{u_{1}}}+\frac{1}{\sqrt{u_{1}+u_{2}}}=\gamma_{1}^{-1} \\
\frac{1}{\sqrt{u_{1}+u_{2}}}=\gamma_{2}^{-1}
\end{array}\right.
$$

The resulting equivalent link capacities are $c_{1}=\left(\gamma_{1}^{-1}-\right.$ $\left.\gamma_{2}^{-1}\right)^{-1}+r_{1}$ and $c_{2}=\gamma_{2}+r_{2}$. Now the problem reduces to that of scheduling, i.e.,

$$
\begin{aligned}
b_{\mathrm{FC}}= & \min q_{1}+q_{2} \\
& q_{1} \geq c_{1}, \\
& q_{2} \geq c_{2}, \\
& q_{1}, q_{2} \geq 0
\end{aligned}
$$

Clearly, the solution is $b_{\mathrm{FC}}=c_{1}+c_{2}$.

The results of the approximations are summarized in the following table.

\begin{tabular}{|l|c|}
\hline Approximation & Value (Mbps) \\
\hline$b_{\mathrm{OPT}}$ & 1.04 \\
\hline$b_{\mathrm{LP}}$ & 1.00 \\
$b_{\mathrm{FS}}$ & 1.10 \\
$b_{\mathrm{FC}}$ & 1.10 \\
\hline
\end{tabular}

\subsubsection{Dimensioning with the Shannon model}

With the Shannon model, the mode matrix is given by

$$
\mathbf{S}=\left(\begin{array}{cc}
c_{1}(p) & 0 \\
0 & c_{2}(p)
\end{array}\right), \quad \begin{aligned}
& c_{1}(p)=W \log _{2}\left(1+\frac{d_{A B}^{-4} p}{\nu_{i}}\right) \\
& c_{2}(p)=W \log _{2}\left(1+\frac{d_{B C}^{-4} p}{\nu_{i}}\right) .
\end{aligned}
$$

The following parameter values are assumed: $d_{A B}=2 \mathrm{~km}$, $d_{B C}=3 \mathrm{~km}, W=3.84 \mathrm{MHz}, \nu_{i}=1.5287 \cdot 10^{-11} \mathrm{~mW}$. The capacity limit of the system is $p_{\mathrm{CL}}=129 \mathrm{~mW}$.

The optimal solution is obtained by solving

$$
\begin{aligned}
p_{\mathrm{OPT}}= & \min _{t, p} p \\
& \Gamma_{1}^{\mathrm{OPT}}\left(\left[t c_{1}(p),(1-t) c_{2}(p)\right]^{\mathrm{T}}\right) \geq \gamma_{1}, \\
& \Gamma_{2}^{\mathrm{OPT}}\left(\left[t c_{1}(p),(1-t) c_{2}(p)\right]^{\mathrm{T}}\right) \geq \gamma_{2} .
\end{aligned}
$$

This is again solved numerically.

The LP approximation gives an optimistic value to the required capacity. The problem is formulated as

$$
\begin{aligned}
f_{\mathrm{LP}}(p)= & \min q_{1}+q_{2} \\
& c_{1}(p) q_{1} \geq r_{1}+\gamma_{1}, \\
& c_{2}(p) q_{2} \geq r_{2}+\gamma_{2}, \\
& q_{1}, q_{2} \geq 0
\end{aligned}
$$

The optimal power is found by line search: $p_{\mathrm{LP}}=\{\min p \geq$ $\left.p_{\mathrm{CL}}: f_{\mathrm{LP}}(p)=1\right\}$.

The optimum of the LP approximation is realized by the schedule $\mathbf{t}^{*}=[0.0958575,0.904142]^{\mathrm{T}}$. We utilize this schedule in the fixed schedule approach (FS), which corresponds to the line search

$$
\begin{aligned}
p_{\mathrm{FS}}= & \min _{p} p \\
& \Gamma_{1}^{\mathrm{ISF}}\left(\mathbf{S}(p) \mathbf{t}^{*}\right) \geq \gamma_{1}, \\
& \Gamma_{2}^{\mathrm{ISF}}\left(\mathbf{S}(p) \mathbf{t}^{*}\right) \geq \gamma_{2}, \\
& p>p_{\mathrm{CL}} .
\end{aligned}
$$

Again, one could have also used the exact throughput formulae $\Gamma_{i}^{\mathrm{OPT}}$.

Finally, we use the fixed capacity vector approach (FC). Recall from the Boolean model case, the equivalent link capacities under the SF bound are given by $c_{1}=\left(\gamma_{1}^{-1}-\right.$ $\left.\gamma_{2}^{-1}\right)^{-1}+r_{1}$ and $c_{2}=\gamma_{2}+r_{2}$. As in the LP approximation approach, we find the power which corresponds to the unit length schedule, i.e., we conduct the line search $p_{\mathrm{FC}}=\left\{\min p \geq p_{\mathrm{CL}}: f_{\mathrm{FC}}(p)=1\right\}$, where

$$
\begin{aligned}
f_{\mathrm{FC}}(p)= & \min q_{1}+q_{2} \\
& c_{1}(p) q_{1} \geq c_{1}, \\
& c_{2}(p) q_{2} \geq c_{2}, \\
& q_{1}, q_{2} \geq 0
\end{aligned}
$$

The results of the approximations are summarized in the following table.

\begin{tabular}{|l|c|}
\hline Approximation & Value $(\mathrm{mW})$ \\
\hline$p_{\mathrm{OPT}}$ & 189 \\
\hline$p_{\mathrm{LP}}$ & 186 \\
$p_{\mathrm{FS}}$ & 210 \\
$p_{\mathrm{FC}}$ & 192 \\
\hline
\end{tabular}

Note that the capacity of link 2 with the computed power values is within the range $0.78-0.87 \mathrm{Mbps}$, which is in good agreement with the results of the Boolean model (1.0$1.1 \mathrm{Mbps})$.

\subsection{Mesh network example}

Next we consider the dimensioning of a realistic scale WMN with multiple access points. The network topology together with the access points (AP) and the used routes (classes) are shown in Figure 3. Note that there are also two relay nodes $(\mathrm{R})$ in the network. Altogether there are 22 links and 18 traffic classes. Also, it is assumed that each node is equipped with a single radio. The offered load on each route is $2 \mathrm{Mbit} / \mathrm{s}$ and the target per flow throughput requirement is $200 \mathrm{kbit} / \mathrm{s}$, i.e., $\rho_{i}=2 \mathrm{Mbit} / \mathrm{s}$ and $\gamma_{i}=200 \mathrm{kbit} / \mathrm{s}$ for all $i$. For the Shannon model the following parameter values are assumed: $\alpha=4, W=10 \mathrm{MHz}$ and $\nu_{i}=3.9811 \cdot 10^{-11}$ $\mathrm{mW}$. The capacity limits of the system are $b_{\mathrm{CL}}=36 \mathrm{Mbit} / \mathrm{s}$ and $p_{\mathrm{CL}}=350 \mathrm{~mW}$ for the Boolean and the Shannon model, respectively.

In this case, the scale of the problem is so large that the joint optimization of the schedule and the capacity parameters becomes numerically difficult. Thus, we only consider the approximate methods of Section 4, i.e., the LP approximation (LP), the fixed schedule approach (FS) and the fixed capacity approach (FC). In the FS approach, the schedule is taken from the LP approximation problem and the capacity parameter is then scaled to meet the ISF bound. The results for the Boolean model and the Shannon model are given in the following table. 


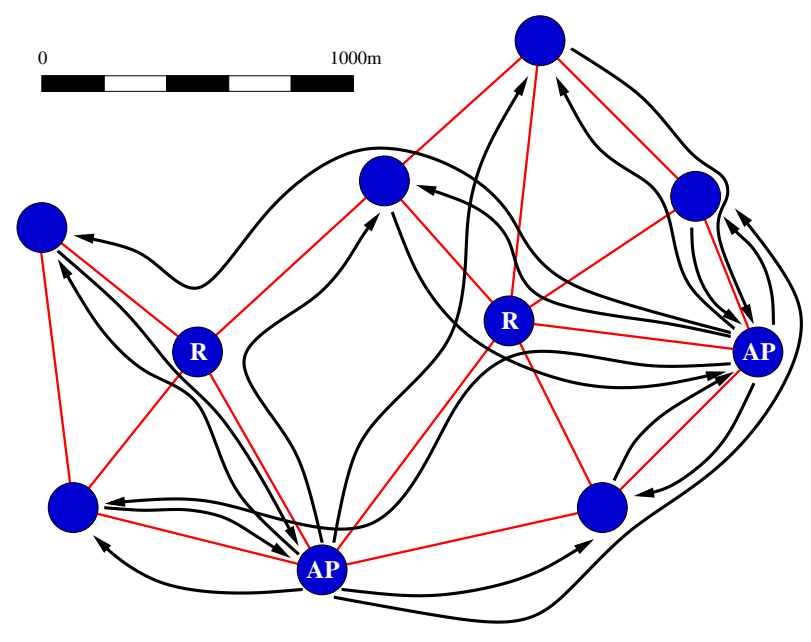

Figure 3: Mesh network topology and the routes in the network.

\begin{tabular}{|c|c|c|}
\hline Approximation & Boolean (Mbps) & Shannon (mW) \\
\hline LP & 38.2 & 500 \\
FS & 41.1 & 1390 \\
FC & 41.8 & 860 \\
\hline
\end{tabular}

The above results emphasize the problems of the FS approach in the Shannon model. Even though, in this case, a result is obtained, it is significantly greater than the results of the other approaches. As discussed earlier, this is due to the way the link capacities depend on the transmission power.

\section{CONCLUSIONS}

We have studied the dimensioning of WMN based access networks with elastic traffic subject to flow-level performance constraints. We have formulated the dimensioning problems for two capacity parameters resulting from different interference models in order to provide order-ofmagnitude estimates of the required transmission resources in such networks. Furthermore, three approximation schemes have been presented to facilitate the computations. The results can be used to evaluate the feasibility and sensitivity of any network scenario defined in terms of node locations and traffic load matrix.

The LP approximation utilizes an upper bound for the flow throughput. For the Boolean model the approach results in an especially appealing form as the entire dimensioning problem can be expressed as a single LP problem. In general, the LP approximation gives an estimate of the necessary amount of required resources. The fixed schedule and fixed capacity vector approaches apply lower bounds for the throughput thus providing a conservative estimate of the required resources. Together the three approaches effectively characterize the capacity parameter.

The assumption of STDMA scheduling allows a very versatile modeling of wireless interference. However, a significant constraint in the modeling is the quickly growing number of different transmission modes. One direction for future research is to apply more elaborate computational methods along the lines of, e.g., [1] to allow larger networks to be dimensioned. Another direction is to address the extensions of the network model. Since in this paper we have considered only elastic traffic, an important extension to the current model is to incorporate requirements for real-time traffic, such as the packet delay of VoIP traffic. Also our assumption of predetermined routes could be relaxed by embedding the routing problem in the dimensioning task and perhaps even allow the use of multi-path routes.

\section{REFERENCES}

[1] P. Björklund, P. Värbrand, and D. Yuan. Resource optimization of spatial TDMA in ad hoc radio networks: a column generation approach. In Proceedings of IEEE INFOCOM, pages 818 - 824, April 2003.

[2] T. Bonald. Throughput performance in networks with linear constraints. In Proceedings of 40 th Annual Conference on Information Sciences and Systems (CISS), Mar. 2006.

[3] T. Bonald, L. Massoulié, A. Proutière, and J. Virtamo. A queueing analysis of max-min fairness, proportional fairness and balanced fairness. Queueing Systems, 53:65-84, 2006.

[4] T. Bonald, P. Olivier, and J. Roberts. Dimensioning high speed IP access networks. In Proceedings of ITC-18, pages 241-251, Aug. 2003.

[5] T. Bonald and A. Proutière. Insensitive bandwidth sharing in data networks. Queuing Systems, 44:69-100, 2003.

[6] T. Bonald and A. Proutière. On performance bounds for balanced fairness. Performance Evaluation, 55:25-50, 2004.

[7] T. Bonald, A. Proutière, J. Roberts, and J. Virtamo. Computational aspects of balanced fairness. In Proceedings of 18th International Teletraffic Congress, pages 801-810, Aug. 2003.

[8] T. Bonald and J. Virtamo. Calculating the flow level performance of balanced fairness in tree networks. Performance Evaluation, 58:1-14, 2004

[9] R. Bruno, M. Conti, and E. Gregori. Mesh networks: commodity multihop ad hoc networks. IEEE Communications Magazine, 43(3):123-131, 2005.

[10] M. Grossglauser and D. Tse. Mobility increases the capacity of ad hoc wireless networks. IEEE/ACM Transactions on Networking, 10(4):477-486, Aug. 2002.

[11] P. Gupta and P. R. Kumar. The capacity of wireless networks. IEEE Transactions on Information Theory, 46(2):388-404, Mar. 2000.

[12] IEEE. IEEE standard for local and metropolitan area networks part 16: Air interface for fixed broadband wireless access systems, 2004. IEEE Std 802.16-2004 (Revision of IEEE Std 802.16-2001).

[13] K. Jain, J. Padhye, V. Padmanabhan, and L. Qiu. Impact of interference on multi-hop wireless network performance. In Proceedings of ACM MobiCom, pages 66-80, Sept. 2003.

[14] M. Johansson and L. Xiao. Cross-layer optimization of wireless networks using nonlinear column generation. IEEE Transactions on Wireless Communications, 5(2):435-445, Feb. 2006.

[15] J. Jun and M. L. Sichitu. The nominal capacity of wireless mesh networks. IEEE Wireless Communications, 10(5):8-14, 2003.

[16] L. Kleinrock. Queueing systems, vol II: Computer applications. John Wiley \& Sons, 1st edition, 1976.

[17] P. Lassila and J. Virtamo. Access network dimensioning for elastic traffic based on flow-level QoS. Technical report, Networking Laboratory, Helsinki University of Technology, June 2005. available at http:/tutkimus/pannet/publ/baldimen.pdf

[18] R. Nelson and L. Kleinrock. Spatial TDMA: A collision-free multihop channel access protocol. IEEE Transactions on Communications, 33(9):934-944, Sept. 1985.

[19] A. Penttinen and J. Virtamo. Performance of wireless ad hoc networks under balanced fairness. In Proceedings of Networking 2004, pages 235 - 246, May 2004.

[20] A. Penttinen, J. Virtamo, and R. Jäntti. Performance analysis in multi-hop radio networks with balanced fair resource sharing. Telecommunications Systems, 31(4):315-336, 2006.

[21] M. Pióro and D. Medhi. Routing, Flow, and Capacity Design in Communication and Computer Networks. Morgan Kaufmann Publishers, 2004.

[22] B. Radunovic and J.-Y. L. Boudec. Optimal power control, scheduling and routing in UWB networks. IEEE Journal on Selected Areas in Communications, 22(7):1252-1270, Sept. 2004. 